\title{
Boosting the Uplink Throughput of OFDM Systems by Creating Resolvable Interference
}

\author{
Manar Mohaisen $\cdot$ Bing Hui $\cdot$ KyungHi Chang
}

\begin{abstract}
Multiple-input multiple-output with orthogonal frequency division multiplexing technology (MIMO-OFDM) is considered to be the ultimate solution for increasing system throughput and for enhancing communication reliability. In this paper, we propose to increase the uplink (UL) throughput by assigning the same UL resources to multiple single-antenna mobile stations. This leads to the loss of orthogonality among sub-carriers. Thus, at the base station (BS), MIMO-OFDM detection techniques are used to separate the streams of different users assigned the same UL resources. To obtain a realistic performance evaluation, different channel scenarios are applied with different correlation values among the antennas of the users. Simulation results show that the proposed MIMO-OFDM system linearly increases the uplink capacity of the OFDM system while maintaining a mobile station transmitter as simple as that used in a conventional OFDM system. For instance, when 4 users are assigned the same UL resources, the throughput of the proposed system is 3.07 times that achieved by a conventional single input single output OFDM system.
\end{abstract}

Key words : Capacity Enhancement, Single-Antenna, MIMO-OFDM Detection, Interference Cancellation.

\section{I . Introduction}

Multiple-input multiple-output (MIMO) and orthogonal frequency division multiplexing (OFDM) technologies are considered to be among the most important technical developments in recent years [1], [2]. MIMO spatial multiplexing boosts the channel throughput without requiring additional spectral resources, while OFDM increases the robustness of the communication system against frequency selective channels. Thus, combining these two technologies is considered to be fundamental for the next generation of communication systems.

Due to space limitations and cost considerations, multiple antennas are not actively installed at a mobile station (MS). Also, it is impractical to implement respectively high complexity signal processing schemes because of the low computational power of MSs. Therefore, in an OFDM system, where MS has only one transmit antenna, none of the MIMO spatial multiplexing techniques can be applied for the uplink (UL) transmission. As a consequence, the UL throughput can't be significantly increased, though some diversity gain can be achieved by the receive antennas of the base station (BS) using linear combiners [3]. Another option for increasing UL throughput is using spatial division multiple accesses (SDMA), where users are spatially identified based on their location [4]. In spite of the appreci- able performance improvement by SDMA, it is always very sensitive to the location of the MSs and requires a high number of antennas to achieve the required performance. Furthermore, the performance achieved by SDMA can be severely degraded when users move rapidly, since the beam-steering information is no longer accurate.

In this paper, we address the issue of increasing the UL throughput of an OFDM system when the MSs use single-antenna transmitters. In order to increase the UL throughput, users are assigned the same frequency and time resources, which leads to a loss in orthogonality among sub-carriers, and consequently the performance is degraded. Therefore, at the BS side, MIMO detection techniques are employed to separate the data streams of each user assigned to the same UL time and frequency resources. By using efficient detection techniques, the effect of the intentionally created interference, i.e., the streams of other users can be removed while almost achieving the performance of a conventional interferencefree OFDM system. MIMO detection literature includes a variety of schemes that differ in terms of the adopted criterion, complexity, and achieved performance. Herein, we use three different detection techniques, namely, zero-forcing (ZF) [5], QRD (QR Decomposition)-based successive interference cancellation (QRD-based SIC) [6], and sphere decoding (SD) [7]. ZF is a linear tech-

Manuscript received March 3, 2011 ; revised May 3, 2011. (ID No. 20110303-008J)

Graduate School of IT \& T, Inha University, Incheon, Korea.

Corresponding Author : KyungHi Chang (e-mail : khchang@inha.ac.kr) 
nique in which the received vector is treated using a filtering matrix based on the ZF criterion. QRD-based SIC detects the transmitted streams successively, where already-detected interference is subtracted out from the received vector and results in a system with less interference. Finally, SD achieves a near-maximum likelihood (ML) performance with a significant reduction in the expected computational complexity.

The proposed system does not require any change in the transmitter structure of the MS. Thus, only the receiver of the BS should be equipped with at least as many antennas as the number of users assigned the same UL resources.

The rest of this paper is organized as follows: Section II presents a detailed description of the proposed system including the transmitter structure of the MS, and the receiver structure of the BS. Furthermore, the complexities of detection techniques are derived. In Section III, we present simulation results and provide and in-depth discussion on the performance of the proposed system under different scenarios. In addition, we show the tremendous throughput increase achieved by the proposed system. Finally, in Section IV, we present our conclusions.

\section{II . System Overview}

\section{2-1 Proposed System Model}

Fig. 1 illustrates the proposed system when three users are assigned the same UL resources. BS allocates the same UL resources to users, with as many uncorrelated channels as possible. This allocation process can be based on the channel estimate at the UL.

Correlation among the single-antennas of the different users has an important effect on the performance of the overall system. Thus, when the correlation is low, the MIMO channel matrix becomes well-conditioned, and the performance of the detection schemes is improved. On the other hand, when the correlation is high, the

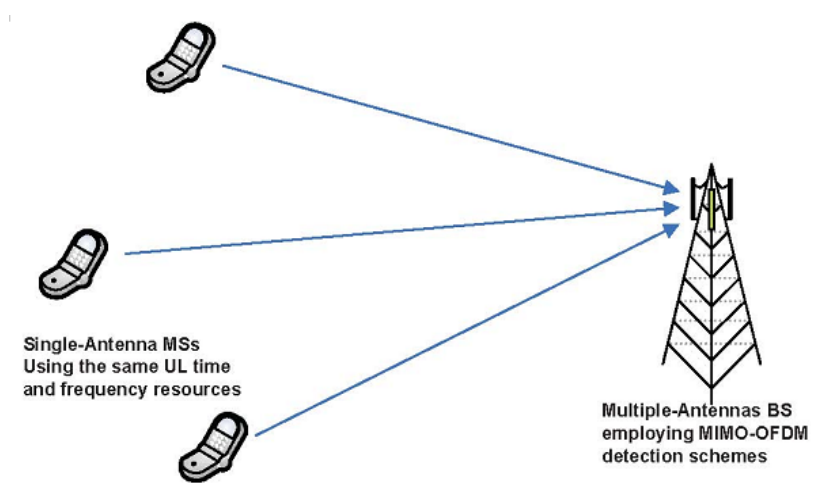

Fig. 1. System model for the proposed system. channel matrix becomes ill-conditioned. As a consequence, the performance of the detection schemes with fixed complexity, e.g., ZF and QRD-based SIC, is degraded and the complexity of the probabilistic schemes, e.g., SD, is increased. Therefore, we examine the performance of the proposed system under different correlation values.

An MS transmitter structure is depicted in Fig. 2. At first, the data stream is encoded using a turbo encoder with a code rate $=1 / 2$. The encoded bits are interleaved using a random interleaver to obtain frequency diversity and are then modulated. The virtual carriers (VC) are inserted, followed by the inverse Fourier transform (IFFT) to obtain the time-domain signal. The guard interval (GI) is then inserted to avoid inter-symbol interference (ISI). Finally, the signal is parallel to the serial converted and transmitted via a single-antenna transmitter.

Fig. 3 depicts the receiver structure of the BS, where multiple antennas are employed. At first, the received signals are demodulated using an OFDM demodulator. A ZF pilot-assisted channel estimate is obtained and is fed to the MIMO detection block. The data streams of the different users are detected and soft LLR values are generated, de-interleaved, and provided to the turbo channel decoder. Finally, a hard decision is applied to recover the data bits at the output of the channel decoder.

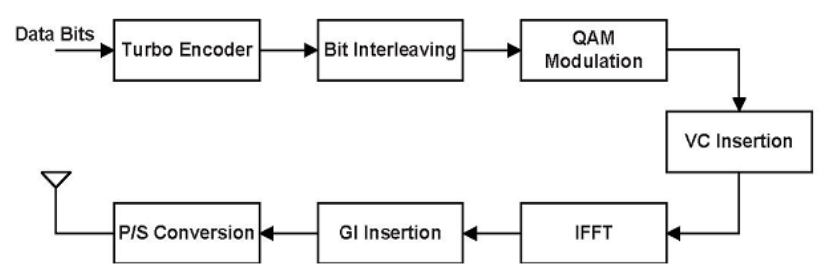

Fig. 2. Transmitter structure of a single-antenna mobile station.

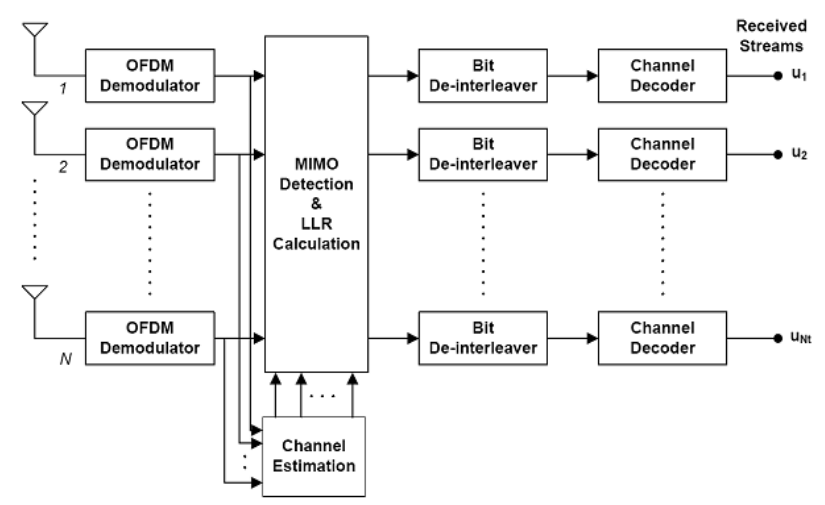

Fig. 3. Receiver structure for a multi-antenna base station. 


\section{2-2 Detection Schemes for a MIMO-OFDM System}

Consider that $N$ MSs are assigned the same resources for their UL transmissions. Therefore, we arrange the transmitted signals in the vector $x \in C^{N}$ in such a way that $x_{j}$ belongs to the $\mathrm{j}$-th user. Furthermore, consider that the number of receive antenna at the BS is equal to $N$. Then at any subcarrier, the baseband received vector $x \in C^{N}$ is given by:

$$
\begin{aligned}
r & =H x+v \\
& =z+v,
\end{aligned}
$$

where $H \in C^{N \times N}$ is the MIMO channel matrix whose element $h_{i-j}$ is the complex transfer function between the $\mathrm{j}$-th user and the $\mathrm{i}$-th receive antenna of the BS. Furthermore, $v \in C^{N}$ is the Gaussian noise drawn from an i.i.d. wide sense stationary process with $E\left[v v^{H}\right]=\delta^{2} I_{N}$. Then, (1) can be considered as the result of perturbing the lattice point $\mathbf{z}$ by the noise vector $\mathbf{v}$.

Let $S=2 N$ denote the dimension of the real Euclidean space $R^{S}$. Then, (1) can be mapped to the real domain by the following equation [8]:

$$
\left[\begin{array}{c}
R(r) \\
I(r)
\end{array}\right]=\left[\begin{array}{cc}
R(H) & -I(H) \\
I(H) & R(H)
\end{array}\right]\left[\begin{array}{c}
R(x) \\
I(x)
\end{array}\right]+\left[\begin{array}{c}
R(v) \\
I(v)
\end{array}\right],
$$

where $R$ and $I$ denote the real and imaginary parts, respectively. Based on (2), we implement the MIMO detection schemes at the receiver side in the real-domain. Equation (2) can be re-written in the real Euclidean space $R^{S}$ as follows:

$$
r_{r}=H_{r} x_{r}+v_{r}
$$

where the subscript $r$ indicates the real-domain.

In the following, we introduce three MIMO detection schemes which representing the main detection categories. In addition, we derive the complexity of these schemes, which is defined as the number of flops required to finish the detection process. Therefore, multiplication, division, and square, and square root operations are counted as one flop for each. On the other hand, we neglect the addition operation because of its respectively low complexity.

\section{2-2-1 Linear Zero Forcing}

$\mathrm{ZF}$ is one of the simplest detection techniques, where received signals are treated using a filtering matrix created based on the $\mathrm{ZF}$ criterion. The filtering matrix is the pseudo-inverse of the channel matrix and is given by $H_{r}^{+}=\left(H_{r}^{T} H_{r}\right)^{-1} H_{r}^{T}$, where $H_{r}^{T}$ is the transpose of matrix $H_{r}$. Linear $\mathrm{ZF}$ does not deploy any sort of ordering; hence, weak received streams are dominated by strong received streams. As a consequence, the performance is degraded.

Based on the assumptions stated earlier, the complexity of $\mathrm{ZF}$ detection is given by:

$$
f_{Z F}=\left(\frac{7}{3} S^{3}+S^{2}-\frac{S}{3}\right)
$$

where the matrix inversion is obtained using the Gaussian elimination method. Furthermore, we considered that the matrix-by-matrix multiplication requires $S^{3}$ flops.

\section{2-2-2 QRD-based Successive Interference Cancellation (QRD-based SIC)}

To reduce the number of interferers in the detection process, Shiu and Kahn [9] proposed decomposing the channel matrix using the QR decomposition (QRD) into the multiplication of a unitary matrix $Q$, and an uppertriangular matrix $R[10]$. In light of the QRD, (1) can be re-written as

$$
y=R x_{r}+n,
$$

where $y=Q^{T} r_{r}$ and $n=Q^{T} v_{r}$. $Q$ has unit-power columns, so, multiplying it to both sides of (1) does not change the noise variance. Furthermore, because of the structure of the matrix $R$, the last component at the bottom of vector $x_{r}$ is interference-free, thus it is the first to be detected. The already-detected component is subtracted out from the received vector and results in a system with fewer interferers. The $(\mathrm{S}-1)$ th component is then detected and cancelled out from the received vector. The process is repeated in a successive way until the first component of the vector $x_{r}$ is detected.

Table 1 illustrates the steps in the QRD-based SIC scheme. Lines (3-7) represent the successive interference cancellation process, where $Q[\cdot]$ is the demodulation of the zero-forcing solution obtained in line (4).

The QRD-based SIC scheme depicted in Table 1 consists of the QR decomposition of the channel matrix, multiplying the vector $r_{r}$ by the matrix $Q^{T}$, and the successive cancellation stage. The QR decomposition is computed by means of householder reflection [11]. Then, the complexity of the QRD-based SIC scheme is given by

$$
\begin{aligned}
f_{Q R D-S I C} & =\sum_{k=1}^{S-1} 2(S-k+1)^{2}+2(S-1)+S^{2}+\sum_{j=1}^{S} j, \\
& =\frac{2}{3} S^{3}+\frac{5}{2} S^{2}+\frac{2}{3} S-4,
\end{aligned}
$$

where the term in the first line of (5) is the cost of multiplying $r_{r}$ by $Q^{T}$.

\section{2-2-3 Sphere Decoding (SD)}

SD was proposed to reduce the complexity of ML de- 
Table 1. QRD-based successive interference cancellation scheme.

\begin{tabular}{|ll|}
\hline Inputs : & $\boldsymbol{H}_{\boldsymbol{r}}, \boldsymbol{r}_{\boldsymbol{r}}$ \\
(1) & $\boldsymbol{H}_{\boldsymbol{r}}=\boldsymbol{Q R}$ \\
(2) & $\boldsymbol{y}=\boldsymbol{Q}^{\boldsymbol{T}} \boldsymbol{r}_{\boldsymbol{r}}$ \\
(3) & For $i=S \ldots \ldots 1$ \\
& \multicolumn{1}{c}{$y_{i}-\sum_{k=i+1}^{N} R_{i, k} x_{k}$} \\
(4) & $\hat{x}_{i}=\frac{R_{i, i}}{}$ \\
(5) & $x_{i}=Q\left[\widehat{x_{i}}\right]$ \\
(6) & $i=i-1$ \\
(7) & End \\
Output : & $\mathbf{x}$ \\
\hline
\end{tabular}

tection while achieving close to the same performance. The SD based on the Schnorr- Euchner strategy was first applied to digital communications by Agrell et al. in [12].

The main idea behind SD is to transform the complex S-dimensional search problem into $S$ parallel 1-dimensional search problems, i.e., search over lines. Furthermore, the search radius can be predefined to reduce the size of the search tree. In other words, instead of trying all the points of the lattice $z$, i.e., ML detection, only those points residing within a sphere of a predefined radius are considered. Thus, SD tests all hypotheses satisfying

$$
\sum_{i=1}^{S}\left|R_{j, j}\left(\hat{x}_{j}-x_{j}\right)+\sum_{i=j+1}^{S} R_{j, i}\left(\hat{x}_{j}-x_{j}\right)\right|^{2} \leq d^{2},
$$

where $R_{j, i}$ is the element of the matrix $R$ located at the $\mathrm{j}$-th row and the $\mathrm{i}$-th column, and $\mathrm{x}$ is the demodulation of the zero-forcing solution $\hat{x}$.

In light of (7), the complexity of SD is variable depending on the signal to noise ratio (SNR), modulation set size, e.g., 4 for QPSK, and the conditioning of the channel matrix. An important work on defining the expected complexity of SD was done by Jalden and Otters- ten in [13]. In this paper, the complexity of SD is obtained using simulation, where we followed the same flops counting methodology explained earlier. The complexity of SD is thus given by

$$
\begin{aligned}
f_{Q R D-S I C} & =\sum_{k=1}^{S-1} 2(S-k+1)^{2}+2(S-1)+S^{2}+A, \\
& =\frac{2}{3} S^{3}+2 S^{2}+\frac{7}{3} S-4+A,
\end{aligned}
$$

where $A$ is the average number of flops required to finish the detection process. Here, $A$ is the only random number of (8), and is obtained using extensive simulations.

\section{2-3 Throughput of the Proposed System}

Communication system throughput can be calculated directly from the channel matrix by considering perfect detection [14]. In this paper, we calculate the system throughput based on the packet error rate (PER) using different detection schemes. In [15], the throughput of the conventional single-carrier communication system is derived. Based on [15], we derived the throughput achieved by proposed system when considering all the influential system parameters. Then, the throughput in bit per second (bps) is given by:

$$
T=\sum_{i=1}^{U}(1-\operatorname{PER}(i))\left[\frac{N_{D S y m b o l}(i)}{T_{s}}\right] C R_{i} \log _{2}\left(M_{i}\right),
$$

where $N_{D \text { Symbol }}$ and $T_{s}$ denote the number of data subcarriers per sub-frame and the sub-frame duration, respectively. $C R$ and $M$ are the code rate of the channel coder and the modulation set size, respectively. Also, $U$ is the number of the single-antenna users assigned the same resources for their UL transmission.

\section{Simulation Results}

\begin{tabular}{|c|c|c|c|c|c|c|}
\hline \multirow[b]{2}{*}{ Path } & \multicolumn{2}{|c|}{ Suburban macro } & \multicolumn{2}{|c|}{ Urban micro } & \multicolumn{2}{|c|}{ Urban micro } \\
\hline & Avg. delay & Path power & Avg. delay & Path power & Avg. delay & Path power \\
\hline 1 & 0.33 & 0.526 & 0.33 & 0.363 & 0.25 & 0.328 \\
\hline 2 & 2.33 & 0.126 & 8.33 & 0.244 & 6.5 & 0.175 \\
\hline 3 & 4.67 & 0.285 & 11.33 & 0.218 & 9.25 & 0.245 \\
\hline 4 & 12.67 & 0.048 & 32.33 & 0.110 & 20.75 & 0.122 \\
\hline 5 & 42.67 & 0.012 & 84.33 & 0.045 & 25.25 & 0.082 \\
\hline 6 & 87.33 & 0.003 & 141.33 & 0.020 & 28.75 & 0.047 \\
\hline
\end{tabular}

\section{3-1 Simulation Environments}

Since wireless communication systems evolve, the need

Table 2. Power and delay profiles of the SCM-E channel for different scenarios. 
to model the MIMO channel becomes urgent. The spatial channel model extended (SCM-E) is one of the best geometric channel models that takes into consideration both the system and environment parameters. The SCME model defines three scenarios; namely, suburban macro, urban macro, and urban micro. The channel is represented by 6 main-paths distinguishable as Dirac delta functions in the delay-domain. Each main-path is comprised of 20 independent sub-paths, where sub-paths are grouped into mid-paths, and each main-path is composed of 3 or 4 mid-paths depending on the channel scenario [16]. Table 2 gives the power and the delay profiles at carrier frequency $f_{c}=3.7 \mathrm{GHz}$ for different scenarios. The delay profile is given as the average number of delay samples of the mid-paths contained in each main-path, and the summation of the power profile values of all the main-paths is equal to 1 .

Fig. 4 depicts the UL frame structure of single-antenna MS when four users are assigned the same resources. The null sub-carriers are reserved for the pilots of the other users to maintain orthogonality among the pilots. Furthermore, when only one user is assigned the UL resources, the null subcarriers are used for data transmission.

Table 3 lists the principal simulation parameters which are adopted mainly from the 3rd Generation Partnership Project Long Term Evolution (3GPP LTE) [17].

\section{3-2 Performance Enhancement by the Proposed System}

The performance of the proposed system is evaluated using different schemes, where four single-antenna MSs are assigned the same time and frequency resources for their UL transmission. Without additional announcement, the distances among users are set to $10 \lambda$ in the simulations. Fig. 5 shows the performance of the proposed system when the packet length is set to 1,200 and 2,400 data bits for QPSK and 16 QAM, respectively.

Linear ZF has the worst performance because of the loss in diversity order, which can be seen from the small slope of the PER curve. The performance degradation, however, is clearer in the case of 16 QAM.

The performance of the proposed system is improved

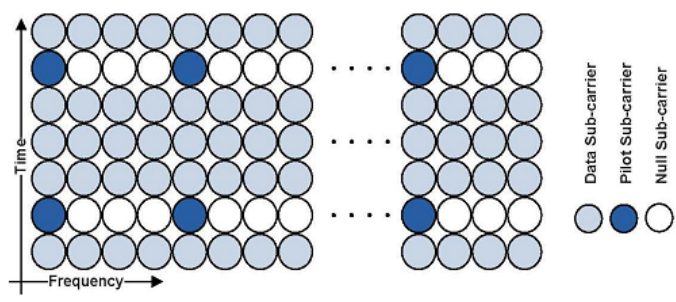

Fig. 4. Uplink frame structure of single-antenna mobile station.
Table 3. Simulation parameters.

\begin{tabular}{|c|c|}
\hline Parameter & Value \\
\hline Bandwidth $(B W)$ & $20 \mathrm{MHz}$ \\
\hline Sampling frequency $\left(f_{s}\right)$ & $30.72 \mathrm{MHz}$ \\
\hline Carrier frequency $\left(f_{c}\right)$ & $3.7 \mathrm{GHz}$ \\
\hline FFT size $\left(N_{F F T}\right)$ & 2,048 \\
\hline $\begin{array}{l}\text { Number of data } \\
\text { subcarriers }\left(N_{D C}\right)\end{array}$ & 1,200 \\
\hline Guard interval $(G I)$ & 146 \\
\hline OFDM sub-frame $\left(T_{s}\right)$ & 7 OFDM symbols $(0.5 \mathrm{~ms})$ \\
\hline Modulation & QPSK, 16 QAM \\
\hline MIMO channel model & $\begin{array}{l}\text { 3GPP spatial channel model } \\
\text { extended (SCM-E) }\end{array}$ \\
\hline Channel type & $\begin{array}{c}\text { Suburban macro, urban macro, } \\
\text { urban micro }\end{array}$ \\
\hline Mobility & $120 \mathrm{~km} / \mathrm{h}$ \\
\hline Channel estimation & Pilot-assisted zero-forcing \\
\hline $\begin{array}{l}\text { BS number of Rx } \\
\text { antennas }\end{array}$ & 1,4 \\
\hline $\begin{array}{l}\text { Number of single-antenna } \\
\text { users }\end{array}$ & 1,4 \\
\hline
\end{tabular}

when the QRD-based SIC scheme is used. QRD-based SIC achieves almost the same diversity order of SD, i.e., same slope of the PER curve, with $6 \mathrm{~dB}$ of performance lagging due to the non-optimality of the adopted decoding strategy. Also, as the modulation order increases, the co-antenna interference (CAI) becomes more effective, consequently the performance is degraded.

Fig. 6(a) depicts the complexity of the detection process using the previously mentioned detection schemes. For the complexity analysis, we considered four single- antenna users and one 4 receive antennas BS. Then, when

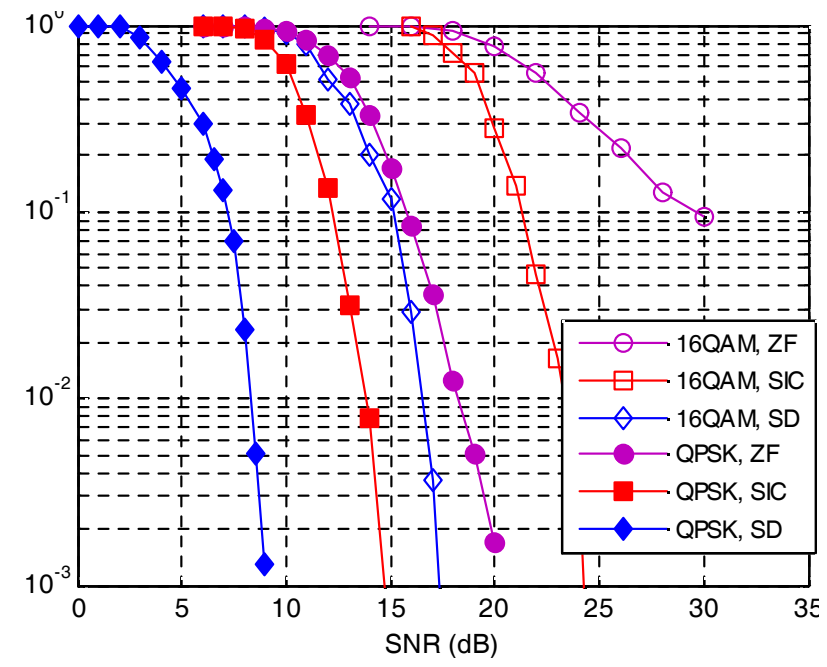

Fig. 5. PER performance of the proposed system for different detection schemes. 


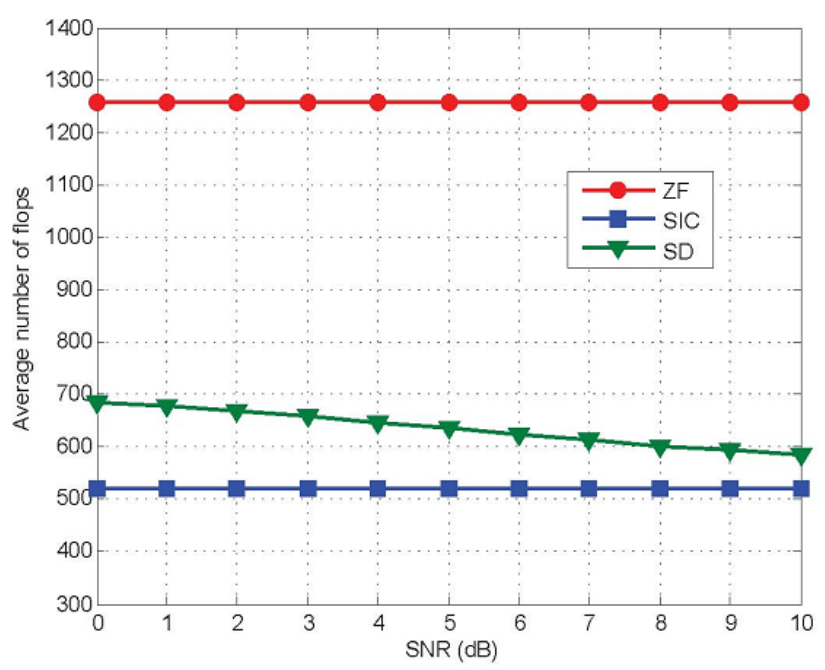

(a) QPSK modulation

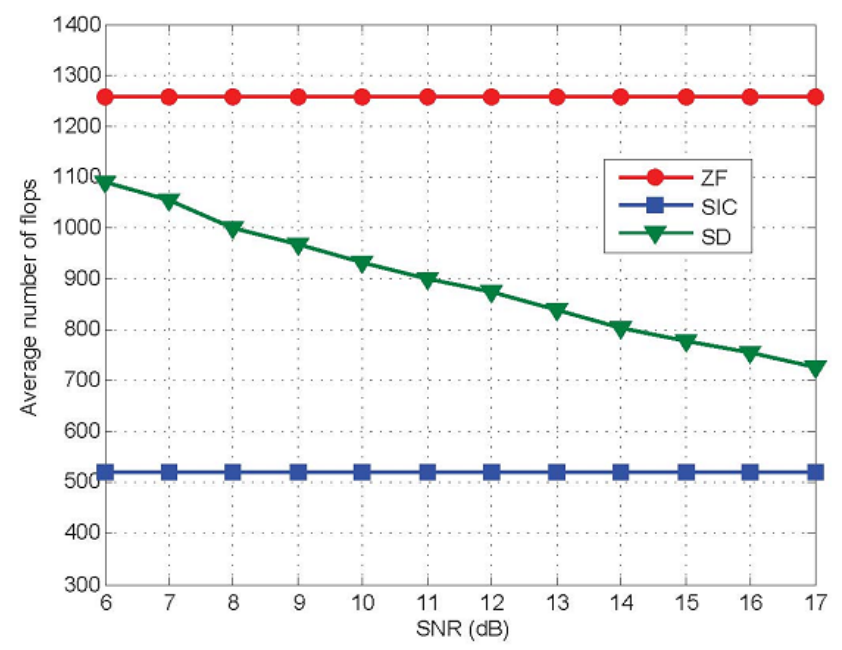

(b) 16 QAM modulation

Fig. 6. Complexity of the proposed system with different detection schemes.

QPSK modulation is used, the expected complexity of $\mathrm{SD}$ is $122 \%$ and $50 \%$ with respect to the complexities of QRD-based SIC and ZF schemes, respectively.

On the other hand, when 16 QAM is used, the size of the lattice increases, and the complexity of the SD scheme also increases, while the complexity of the other two detection schemes remains fixed as shown in Fig. 6(b). The complexity of SD has been an issue in many studies, and other alternative schemes were proposed to reduce its complexity while achieving the same performance [18], [19].

Fig. 7 shows the throughput of the proposed system obtained using (9), for different scenarios. For the sub-frame structure of Fig. 5, the error-free throughput of the conventional SISO OFDM system is $15.6 \mathrm{Mbps}$ and 31.2 Mbps for QPSK and 16 QAM modulated-data, respectively.

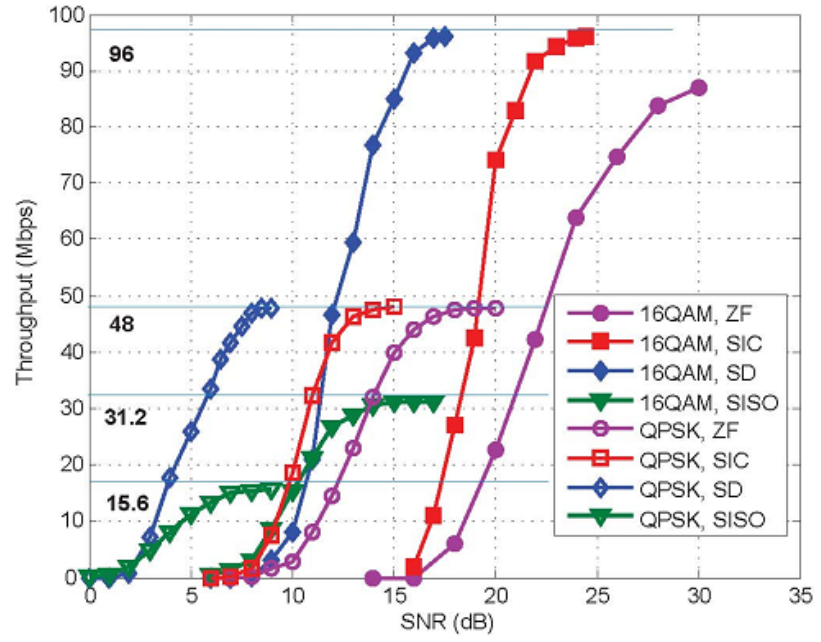

Fig. 7. Throughput of the proposed system, when four users are assigned to the same UL resources.

The proposed system with SD scheme achieves 48 Mbps for QPSK modulation without any additional power requirements, i.e., at the same average SNR. QRDbased SIC, which is indicated as SIC, and ZF attain the $48 \mathrm{Mbps}$ limit at SNR $15 \mathrm{~dB}$ and $20 \mathrm{~dB}$, respectively. In the case of 16 QAM, the proposed system achieved the maximum throughput of $96 \mathrm{Mbps}$, which is 3.07 times that achieved by the SISO system, for both the SD and QRD-based SIC schemes.

In the case of $\mathrm{ZF}$, the maximum throughput at SNR $30 \mathrm{~dB}$ is $88 \mathrm{Mbps}$ because of the performance degradation caused by the use of linear filtering. Also, ZF throughput does not follow the capacity curve, which is due to the loss in the diversity gain.

The performance of the proposed system is affected by the correlation among the antennas of the different users. Fig. 8 shows the PER performance of the proposed system for different physical separations among users with QPSK modulation. In all scenarios, the distance between the antennas of the BS is kept at $10 \lambda$, which is equivalent to $0.81 \mathrm{~m}$. The distance among users has three different values; specifically, $10 \lambda, 500 \lambda$, and drawn from a uniform random variable in a range between $10 \lambda$ and $500 \lambda$. When the distance among users is small, transmit antennas become more correlated, and consequently CAI appears. As shown in Fig. 8, the worst performance is obtained when the distance among users is as short as $10 \lambda$, while the best performance is attained when the distance among users is $500 \lambda$. On the other hand, when the separation among users follows a uniform distribution in a range between $10 \lambda$ and $500 \lambda$, the proposed system performance is degraded by $0.5 \mathrm{~dB}$ as compared with that achieved when the separation is fixed to $500 \lambda$. 


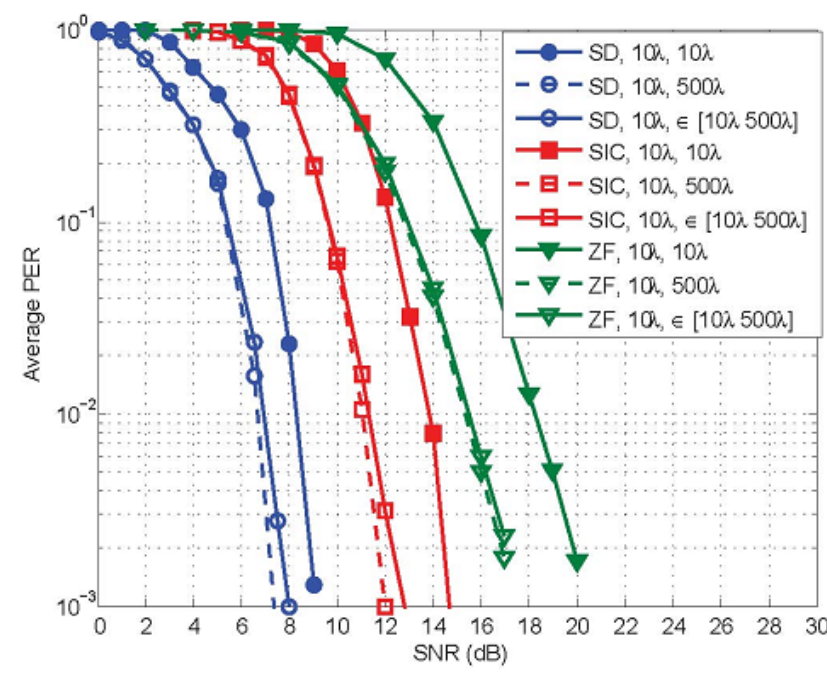

Fig. 8. Effect of the correlation on the performance of the proposed system.

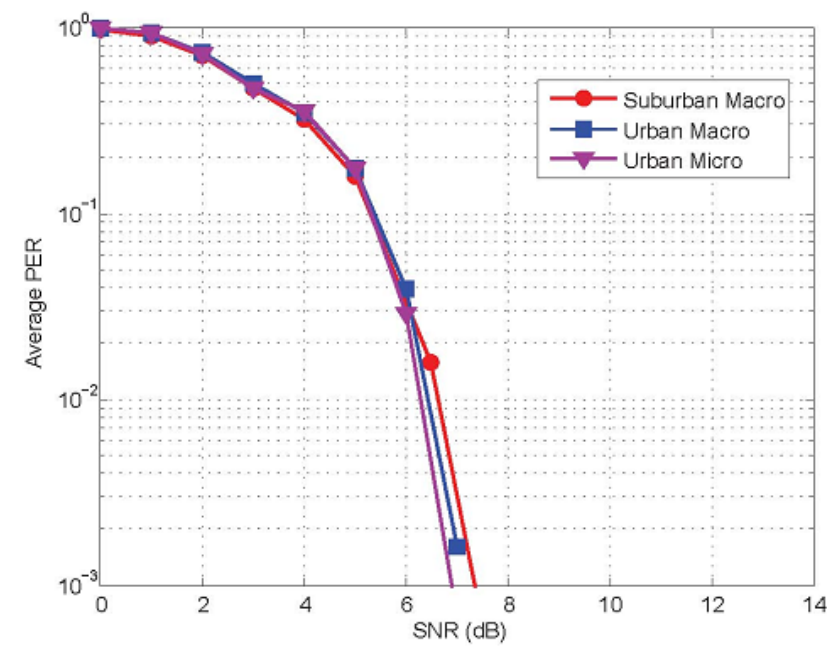

Fig. 9. Performance of the proposed system under different SCM-E scenarios.

Finally, Fig. 9 shows the performance of the proposed system under the three different scenarios of the SCM-E channel. Here, we consider that data are modulated using QPSK and detected using the SD scheme. The best performance of the proposed system is achieved under an urban micro environment. At target PER $10^{-2}$, the performance of the proposed system is degraded by 0.2 $\mathrm{dB}$ and $0.5 \mathrm{~dB}$ under urban macro and suburban macro environments, respectively.

\section{Conclusions}

MIMO spatial multiplexing techniques continue to fascinate researchers for their capabilities to increase system throughput without the need for additional spec- tral resources. In this paper, the throughput of an OFDM system is increased by allocating the same time and frequency resources to different single-antenna users for their UL transmissions. As a consequence, the orthogonality among subcarriers is destroyed, and the performance is degraded. To cancel out the created interference, i.e., streams of other users, MIMO-OFDM detection schemes are employed at the BS. The performance of the proposed system is then evaluated with different detection schemes, and the overall system throughput is calculated. When four users are assigned the same UL time and frequency resources, the throughput of the proposed system is 3.07 times that achieved by the conventional SISO OFDM system using the same modulation schemes. Furthermore, the proposed system shows considerable stability under different channel environments and for different separations among users.

This work was supported by the National Research Foundation of Korea (NRF) grant funded by the Korea government (MEST) (No. R01-2008-000-20333-0).

\section{References}

[1] A. Paulraj, R. Nabaret, and D. Gore, Introduction to Space-Time Wireless Communications, Cambridge Univ. Press: Cambridge, 2003.

[2] R. Van Nee, R. Prasard, OFDM for Wireless Multimedia Communications, Artech House: New York, 2000.

[3] J. Lee, L. Miller, CDMA Systems Engineering Handbook, Artech House: New York, 1998.

[4] A. El Zooghby, Smart Antenna Engineering, Artech House: New York, 2005.

[5] Q. Spencer, C. Peel, A. Swindlehurst, and M. Haardt, "An introduction to the multi-user MIMO downlink," IEEE Communications Magazine, vol. 42, no. 10, pp. 60-67, 2004.

[6] D. Wubbenm, K. -D. Kammeyer, "Low complexity successive interference cancellation for MIMO-OFDM systems," European Trans. on Telecom, vol. 18, pp. 457-466, 2007.

[7] B. Hockwold, S. ten Brink, "Achieving near-capacity on multiple-antenna channel," IEEE Trans. on Commun, vol. 51, no. 3, pp. 389-399, 2003.

[8] H. Vikalo, B. Hassibi, and T. Kailath, "Iterative decoding for MIMO channels via modified sphere decoding," IEEE Trans. on Wireless Commun, vol. 3, no. 6, pp. 2299-2311, 2004.

[9] D. Shiu, J. Kahn, "Layered space-time codes for wireless communications using multiple transmit an- 
tennas," in Proc. Intern. Conf. of Commun., pp. 436440, 1999.

[10] G. Golub, C. Van Loan, Matrix Computations, The Johns Hopkins Univ. Press, 1996.

[11] J. Gentle, Matrix Algebra: Theory, Computations, and Applications in Statistics, Springer, 2007.

[12] E. Agrell, T. Eriksson, A. Vardy, and K. Zeger, "Closest point search in lattices," IEEE Trans. on Inf. Theory, vol. 48, no. 8, pp. 2201-2214, 2002.

[13] J. Jalden, B. Ottersten, "On the complexity of sphere decoding in digital communications," IEEE Trans. on Signal Processing, vol. 53, no. 4, pp. 1474-1484, 2005.

[14] S. Haykin, M. Moher, Modern Wireless Communications, Pearson Prentice Hall, 2005.

[15] J. Andrews, A. Ghosh, and R. Muhamed, Fundamentals of WiMAX: Understanding Broadband Wireless Networking, Pearson Education, 2007.

Manar Mohaisen

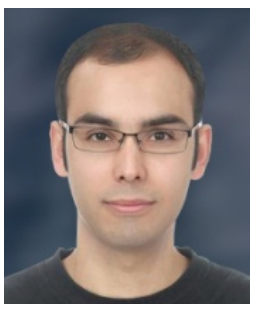

received his B.Eng. in electrical engineering from the University of Gaza (IUG), Gaza, Palestine, in 2001. From 2001 to 2004, he was with the Palestinian Telecommunications Company JAWWAL, Gaza, Palestine, where he worked as an operation and maintenance engineer and then as a cell-planning engineer. $\mathrm{He}$ received his M.S. degree in communication and signal processing from the University of Nice-Sophia Antiplois, Sophia Antipolis, France, in 2005. From March to September 2005, he followed an internship at IMRA Europe Co., Sophia Antipolis, France, as a part of his M.S. degree, where he worked on noise reduction in car environments. In February 2010, he obtained a Ph.D. degree from the Graduate School of Information Technology and Telecommunication, Inha University, Incheon, Korea. Since September 2010, he is with the School of Information Technology Engineering, Korea University of Technology and Education (KUT), where he is an assistant professor. His research interests include 3GPP LTE systems, detection schemes for spatial multiplexing MIMO systems, and precoding techniques for multiuser MIMO systems.
[16] D. S. Baum, J. Salo, G. Del Galdo, M. Milojevic, P. Kyösti, and J. Hansen, "An interim channel model for beyond-3G systems," in Proc. IEEE $V T C$, vol. 5, pp. 3132-3136, May 2005.

[17] 3GPP TR 25.814, "Physical layer aspects for evolved universal terrestrial radio access (UTRA)," v.7.1.0, Sep. 2006.

[18] Y. Morishige, M. Fujii, M. Itami, and K. Itoh, "Tree search detection based on LLR using $\mathrm{M}$ algorithm in MC-CDMA systems," IEICE Trans. Fundamentals, vol. E89-A(10), pp. 2622-2629, 2006.

[19] S. Sun, Y. Dai, Z. Lei, H. Kenichi, and H. Kawai, "Pseudo-inverse MMSE-based QRD-M algorithm for MIMOOFDM," in Proc. of IEEE VTC, vol. 3, pp. 1545-1549, May 2006.

Bing Hui

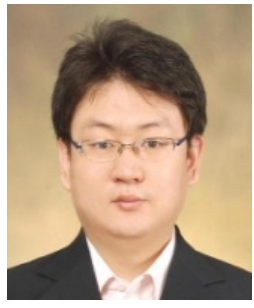

received his B.S. degree in communication engineering from Northeastern University, Shenyang, China, in 2005. He received a M.Eng. degree at the Graduate School of Information Technology and Telecommunications, Inha University, Incheon, Korea, in 2009. From 2007 to 2010, he has been involved in the R\&D projects, whose topics are MIMO-OFDM algorithm including detection, and dirty paper coding type of precoding techniques. Since 2009, he is working as a Ph.D. student in the Graduate School of Information Technology and Telecommunications, Inha University, Incheon, Korea. His research interests include WiMAX and the 3GPP LTE systems, precoding and detection schemes for MIMO systems, interference mitigation techniques in cellular network, optimal codebook design with limited feedback, and mobile ad-hoc networks. 


\section{KyungHi Chang}

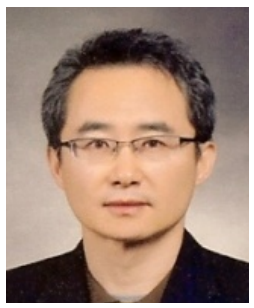

received his B.S. and M.S. degrees in electronics engineering from Yonsei University, Seoul, Korea, in 1985 and 1987, respectively. He received his Ph.D. degree in electrical engineering from Texas A\&M University, College Station, Texas, in 1992. From 1989 to 1990, he was with the Samsung Advanced Institute of Technology (SAIT) as a member of research staff and was involved in digital signal processing system design. From 1992 to 2003, he was with the Electronics and Telecommunications Research Institute (ETRI) as a principal member of technical staff. During this period, he led the design teams working on the WCDMA UE modem and 4G radio transmission technology (RTT). He is currently with the Department of Electronic Engineering, Inha University, where he has been a professor since 2003. His current research interests include RTT design for IMT-Advanced system, 3GPP LTE and M-WiMAX system design, cognitive radio, cross-layer design, cooperative relaying systems, RFID/USN, and mobile ad-hoc networks. Dr. Chang has served as a senior member of IEEE since 1998, and as an editor-in-chief of the Korean Institute of Communication Sciences (KICS) proceedings during 2007 2009. Currently, he is an editor-in-chief of the KICS Journal A. He has also served as an editor of ITU-R TG8/1 IMT.MOD, and he is currently an international IT standardization expert of the Telecommunications Technology Association (TTA). He is an recipient of the LG Academic Awards (2006), Haedong Best Paper Awards (2007), IEEE ComSoc Best Paper Awards (2008), and Haedong Academic Awards (2010). 